\title{
Excess Gestational Weight Gain in Pregnancy and the Role of Lifestyle Intervention
}

\author{
Rebecca Goldstein, MBBS (Hons), FRACP ${ }^{1,2}$ Helena Teede, MBBS, FRACP, PhD ${ }^{1,2}$ \\ Shakila Thangaratinam, MBBS, MRCOG, $\mathrm{PhD}^{3}$ Jacqueline Boyle, MBBS, FRANZCOG, MPH, PhD ${ }^{1,4}$
}

\footnotetext{
${ }^{1}$ Monash Centre for Health Research and Implementation, School of Public Health and Preventive Medicine, Monash University, Victoria, Australia

${ }^{2}$ Diabetes and Endocrinology Units, Monash Health, Monash Medical Centre, Clayton, Victoria, Australia

${ }^{3}$ Women's Health Research Unit, Barts and the London School of Medicine and Dentistry, Queen Mary University of London, London, United Kingdom

${ }^{4}$ Obstetrics and Gynaecology Unit, Monash Health, Clayton, Victoria, Australia
}

\begin{abstract}
Address for correspondence Helena Teede, MBBS, FRACP, PhD, Monash Centre for Health Research and Implementation, School of Public Health and Preventive Medicine, Monash University, Diabetes and Endocrinology Units, Monash Health, Monash Medical Centre Clayton, Melbourne Victoria 3168, Australia (e-mail: helena.teede@monash.edu).
\end{abstract}

Semin Reprod Med 2016;34:e14-e21.

\begin{abstract}
Keywords

- gestational weight gain (GWG)

- outcomes

- intervention

- body mass index (BMI)

With increasingly adverse lifestyles, young women in many countries have rapid weight gain and rising obesity. In keeping with this, most pregnant women exceed recommended gestational weight gain (GWG) and then retain weight postpartum. The consequences of excess GWG include maternal risks during pregnancy, neonatal risks and maternal obesity and chronic disease longer term, presenting a significant public health and economic burden worldwide. This article discusses the adverse maternal and infant risks with excess GWG apparent from observational studies, summarizes the existing guidelines for optimal GWG and highlights the need for further research to identify optimal GWG recommendations across the different ethnicities and weight ranges.

We also review the evidence for lifestyle interventions in pregnancy to prevent excess GWG and highlighting the work underway to integrate large scale meta-analyses of individual patient data from lifestyle intervention studies to inform clinical practice beyond current observational data. Finally, we address the need to implement lifestyle interventions into routine pregnancy care to improve short and long term maternal health outcomes.
\end{abstract}

Obesity secondary to adverse lifestyle presents a major public health and economic burden worldwide. Established obesity requires intensive, multidisciplinary and costly treatment. Once obesity is established, lifestyle induced weight loss is largely unsustainable due to physiological adaptation which drives weight regain. ${ }^{1,2}$ In contrast, prevention of weight gain is feasible with minor lifestyle changes ${ }^{3,4}$ and small energy balance adjustments ( $\sim 220 \mathrm{~kJ} /$ day $){ }^{3}$ conveying long term health benefits. In this context, the World Health Organization (WHO) global strategy for the prevention of non-com- municable diseases notes obesity as a preventable condition and recommends to aim to increase physical activity and improve diet ${ }^{5}$ to prevent obesity. This is therefore now a high priority internationally. ${ }^{6}$

The health implications of weight gain are major and the risks increase with each $\mathrm{kg}$ gained across all weight categories, making prevention a priority for all women. Diabetes risk increases above BMI of $22 \mathrm{~kg} / \mathrm{m}^{2}$ with $18 \%$ affected in normal weight, $35 \%$ in overweight and $75 \%$ in obese women. ${ }^{7}$ Cardiovascular disease is the number one cause of mortality from
Issue Theme Lifestyle in Reproductive Medicine; Guest Editors, Lisa Moran, PhD, BND, BSc (Hons) and Helena Teede, MBBS, PhD, FRACP
Copyright $\odot 2016$ by Thieme Medical Publishers, Inc., 333 Seventh Avenue, New York, NY 10001, USA. Tel: +1(212) 584-4662.
DOI http://dx.doi.org/ $10.1055 / \mathrm{s}-0036-1583531$. ISSN 1526-8004. 
non-communicable disease in women and increases by $3 \%$ for each kilo gained. ${ }^{8}$ As $55 \%$ of deaths are lifestyle or weight related, the imperative for effective obesity prevention interventions is critical. $^{9}$

Pregnancy is a key driver of weight gain, with most women in developed countries exceeding recommended gestational weight gain (GWG) with a mean $2-5 \mathrm{~kg}$ retained per pregnancy. ${ }^{10-13}$ Excess GWG is directly related to long-term obesity across all weight categories. ${ }^{14}$ Longitudinal data shows a 300\% increase in obesity risk long term if GWG exceeds guidelines. ${ }^{12}$ Excess GWG thus drives long term obesity and chronic disease. ${ }^{12}$ Pregnancy therefore offers significant opportunities for obesity prevention ${ }^{6}$ and reproductive aged women are now targeted as a high risk group with recommendations to limit GWG and encourage postpartum weight loss. ${ }^{15}$

In additional to driving maternal obesity, excess GWG also worsens pregnancy complications for both mothers and babies. ${ }^{16}$ GWG is an independent predictor of large for gestational age babies and related complications. ${ }^{17}$ The Institute of Medicine (IOM) in the USA has made recommendations for optimal $\mathrm{GWG}^{18}$ based on maternal BMI, although the observational cohorts underpinning these recommendations were from the USA in 1980 with limited obesity and little ethnic diversity.

With regards to lifestyle interventions, leading researchers in the field and the WHO have recognized that the environmental and societal factors driving obesity must be addressed for effective widespread obesity prevention. ${ }^{19,20}$ However environmental and societal changes have proven slow to change with WHO noting that obesity is one of today's most blatantly visible - yet most neglected - public health problems. Ultimately however, individual behavior change, ideally enabled by societal and environmental change is needed to prevent and manage obesity. The evidence on individually targeted lifestyle initiatives is reviewed here, while acknowledging the need for concomitant broader societal and environmental approaches to support individual lifestyle change. ${ }^{20}$

There is currently no literature on healthy lifestyle interventions preconception, despite education opportunities in this life phase. Adopting healthy lifestyle interventions in pregnancy with positive changes to dietary intake and physical activity prevents excess GWG gain and may reduce pregnancy complications based on systematic review and meta-analysis. ${ }^{21}$ They do not impact on birth weight, or result in safety concerns for the fetus. ${ }^{21}$ Factors associated with intervention success include focusing on diet or combined interventions, using behavioral strategies and using technology to support delivery. ${ }^{21-24}$ However, despite the clear health needs and evidence for the efficacy of lifestyle interventions in pregnancy, a major gap persists with inadequate translation of healthy lifestyle change integrated into routine preconception and antenatal care.

Barriers to incorporating a focus on healthy lifestyle behaviors into pregnancy care include women's limited knowledge and awareness of healthy GWG and adverse effects of excess GWG on long term health; inadequate health professional skills, support and training ${ }^{25}$; sociocultural challenges for health professionals and women, limited accessibility of evidence based programs and resources, inadequate staff time and health system challenges. ${ }^{26}$ Enablers include the "teachable moment" with pregnant women more motivated to accept healthy behaviors in pregnancy ${ }^{26-29}$ for the health of their child, and health system engagement with frequent antenatal visits. However, there is inadequate existing implementation research addressing these barriers and leveraging off these enablers.

Currently, extensive international individual patient data meta-analysis of lifestyle interventions in pregnancy is underway and this data will enable us to answer key questions on the efficacy of lifestyle interventions, including diet, physical activity and mixed interventions in pregnancy for prevention of GWG. ${ }^{30}$ It will also allow exploration of lifestyle intervention impact on maternal and neonatal outcomes, and their relative efficacy across the BMI range and different ethnicities.

Here, we consider opportunities for education on diet and lifestyle preconception. We then review the relevant literature on the adverse health outcomes of excess maternal GWG and recommendations for optimal GWG and controversies around existing Institute of Medicine Guidelines for GWG. We also review the literature on lifestyle interventions in pregnancy and outline the pending large scale international individual patient data meta-analysis in this area. We then close with a discussion around next steps toward implementation of healthy lifestyle into routine pregnancy care to prevent excess gestational weight gain.

\section{Preconception Intervention Opportunities to Prevent Weight Gain}

Most women do not engage in maternity care until late in their first trimester. Therefore, pre-conception offers an opportune time for screening for risk factors that may impact on fertility, pregnancy and the future child. There is no evidence from randomized controlled trials (RCT) to support specific interventions or specific models of care pre-conception to improve pregnancy outcomes in overweight or obese women. ${ }^{31}$ However, the preconception period provides an opportunity to assess for and manage weight associated maternal chronic conditions including diabetes, hypertension, sleep apnoea and polycystic ovary syndrome. ${ }^{32-34}$ It is also an ideal time to discuss and offer an individualized diet and physical activity as weight loss pre-conception will improve fertility and pregnancy outcomes in overweight or obese women. Diet and physical activity pre-conception may also improve GWG; a community based RCT of a 6 group session intervention on physical activity, diet, stress and health behaviors pre-conception and inter-conception showed changes in diet, self-efficacy and reported physical activity preconception. ${ }^{35}$ They then followed up women and assessed them across BMI categories; those who were in the intervention group had lower BMI at 12 month follow up and a trend to lower pregnancy GWG after adjustment for pre-pregnancy BMI. ${ }^{36}$ Increased levels of physical activity 
pre-conception are also associated with trends in decreased GWG. ${ }^{37}$ Preconception lifestyle interventions also have the potential to limit first trimester GWG. Until further evidence emerges on the role of preconception interventions for limiting excess GWG, as per National Centre for Clinical Excellence guidelines, addressing chronic conditions and lifestyle factors related to weight in overweight and obese women should be considered. ${ }^{38}$

\section{Adverse Health Outcomes of Excess Maternal GWG: Observational Data}

In the US, Europe and Australia, 20-50\% of women gain more than the recommended GWG during pregnancy. ${ }^{39}$ GWG has major implications in pregnancy, independent of maternal obesity, with every kilo above recommended, linked to $10 \%$ increase in adverse outcomes. ${ }^{16}$ The combination of excess GWG and obesity is concerning and preventing excess GWG across all BMI categories is imperative.

Excess GWG drives some well-recognized short-term adverse maternal outcomes reported in population-based cohort studies (-Table 1), including pre-term birth ${ }^{13,40}$ and caesarean section. ${ }^{13,40-44}$ Other outcomes are more debated, including gestational diabetes (GDM) $)^{17,45}$ and gestational hypertension/pre-eclampsia. ${ }^{42,44}$ Short-term infant outcomes include increased birth weight, ${ }^{13,40,42,44,46}$ LGA and reduced risk for SGA. ${ }^{13,40,41,43,46}$

In the long term, excess GWG increases maternal postpartum weight retention at six, ${ }^{43}$ twelve months ${ }^{41}$ and eighteen months ${ }^{43}$ and predicts long-term obesity, ${ }^{47,48}$ which in turn indirectly predicts diabetes, heart disease ${ }^{49}$ and chronic disease. ${ }^{48}$ Childhood overweight/obesity is also linked strongly to excess GWG on observational studies. ${ }^{41,50}$ Recent literature has also described the association of increased maternal GWG and an adverse adolescent metabolic profile. ${ }^{51,52}$

It is difficult to make meaningful comparisons of the severity and frequency of these outcomes across the observational studies in this area due to differing classification of BMI and GWG categories, differing outcome definitions, inconsistent control for confounding factors and variable study methods. Refinement of core outcome sets and standard endpoint definitions for research in this area is needed, along with intervention research linked to long term cohort studies to explore health outcomes for mothers and children.

\section{Recommendations for Optimal GWG: Observational Data}

As noted, to create comprehensive guidelines regarding ideal GWG, there should be a consensus on core outcome sets and agreed definitions on core endpoints. Currently, this is lacking and guidelines base recommendations on inconsistently applied and defined outcomes from observational studies. Approaches such as that used by Thangaratinam ${ }^{21}$ with a two round Delphi survey of experienced clinicians to rank outcomes for importance in their meta-analysis of interventions in pregnancy is progressing this area and we look forward to clearly defined core outcomes sets in future.

Healthy GWG is not equal across the BMI spectrum. All guidelines allow greater GWG in women who are underweight at the onset of pregnancy. Nohr ${ }^{43}$ found that underweight women can have high GWG without the consequences of adverse maternal and infant outcomes, hence their weight gain allowance is more generous. Most guidelines recommend lower weight gain for overweight and obese women, and across ethnicities, these women are more likely to exceed recommended weight gain, even though their mean weight gain during pregnancy is less than normal weight women. ${ }^{17,41,53}$

- Table 2 summaries the key guidelines. Presently, the IOM $2009^{13}$ guidelines are most commonly used. They are an updated version from the 1990 guidelines, where the 1990 emphasis was on avoiding the consequences of low GWG rather than high GWG, with respect to infant outcomes only. They differ from the 1990 guidelines because they are based on the WHO cut points for maternal BMI categories and include a new narrow range of GWG for obese women. The 2009 guidelines identified maternal and infant outcomes that were based on the Agency for Healthcare research and Quality (AHRQ) systematic review from $2008^{40}$ and commissioned additional analyses. However the 2009 IOM guidelines still derived recommendations from the same original dataset of US based largely Caucasian women in the 1980's when overweight and obesity in pregnancy was relatively uncommon and GWG was more limited. Infant outcomes were SGA, LGA, preterm birth and childhood obesity. Maternal outcomes selected included postpartum weight retention, caesarean section, GDM, gestational hypertension; however, GDM and gestational hypertension were removed from analysis due to lack of sufficient evidence from methodologically

Table 1 Risks of excess GWG

\begin{tabular}{|l|l|l|}
\hline & Short term & Long term \\
\hline Maternal outcomes & GDM & Post partum weight retention \\
\hline & Pre-eclampsia/ gestational hypertension & Obesity \\
\hline & Pre-term birth & \\
\hline Infant outcomes & C section & \\
\hline & Increased birth weight & Childhood obesity \\
\hline & LGA & \\
\hline & Low risk of SGA & \\
\hline
\end{tabular}


flawed observational studies. Strengths of IOM are that the severity and the frequency of the outcomes have been considered in building the guidelines, which other guidelines have not done.

Cedergren ${ }^{16}$ recommended optimal GWG recommendations based on a large Swedish population-based cohort registry of nearly 300,000 women, almost all of Caucasian origin (-Table 2). Interestingly, selection of outcome variables directly related to maternal GWG and BMI was not the purpose of the study. Rather, the aim was to 'estimate weight gain limits that were associated with significantly decreased risk of the most clinically dangerous situations for the mother and the infant'. Analysis included SGA, LGA, preeclampsia and several short-term maternal and infant complications. Recommendations emerging from this work have a narrower limit for GWG than IOM, across all BMI categories. Of note, the outcomes were not weighted for severity and a number are rare, perhaps limiting usefulness. Moreover, weight gain information was only available in $<40 \%$ of women.

The 1990 IOM and Cedergren recommendations have been directly compared using the New Jersey Pregnancy Risk Assessment Monitoring System (PRAMS) database of over 9000 women. ${ }^{54}$ Using the Cedergren guidelines, the incidence of macrosomia and caesarean delivery was lower, however low birth weight, preterm deliveries and neonatal intensive care admissions occurred more frequently. Ideal GWG was assessed to be between both these sets of recommendations.

The IOM guidelines have also been evaluated in large observational datasets. A German study based on more than 170,000 deliveries ( - Table 2 ) created a model for joint predicted risks of SGA and LGA in relation to GWG and found much wider optimal GWG ranges across the BMI range. ${ }^{55}$ More recently, the Norwegian Mother and Child Cohort

Table 2 Key guidelines for GWG

\begin{tabular}{|c|c|c|c|c|}
\hline Author & Rasmussen (IOM) & Cedergren & Beyerlein & Ee \\
\hline Year & 2009 & 2007 & 2009 & 2014 \\
\hline Country of origin & US & Sweden & Germany & Singapore \\
\hline $\begin{array}{l}\text { Guideline development } \\
\text { based on }\end{array}$ & $\begin{array}{l}\text { Systematic review, } \\
\text { commissioned } \\
\text { reports }\end{array}$ & $\begin{array}{l}\text { Population based } \\
\text { cohort study }\end{array}$ & $\begin{array}{l}\text { population based } \\
\text { cohort study }\end{array}$ & $\begin{array}{l}\text { population } \\
\text { based cohort study }\end{array}$ \\
\hline \multirow{6}{*}{$\begin{array}{l}\text { Maternal outcomes } \\
\text { assessed }\end{array}$} & Caesarean section & Preeclampsia, eclampsia & $\mathrm{N} / \mathrm{A}$ & Caesarean section \\
\hline & $\begin{array}{l}\text { Post partum weight } \\
\text { retention }\end{array}$ & Postpartum hemorrhage & & Vaginal delivery \\
\hline & & Venous complications & & \\
\hline & & Shoulder dystocia & & \\
\hline & & Complications of anesthesia & & \\
\hline & & Stillbirth after 28 weeks & & \\
\hline \multirow[t]{9}{*}{ Infant outcomes assessed } & SGA & SGA & SGA & SGA \\
\hline & LGA & LGA & LGA & LGA \\
\hline & Preterm birth & Birth trauma & & AGA (appropriate for \\
\hline & Childhood obesity & Respiratory disorders & & Gestational age) \\
\hline & & Bacterial sepsis & & \\
\hline & & Haemorrhagic disorders & & \\
\hline & & Convulsions & & \\
\hline & & perinatal death & & \\
\hline & & Apgar score $<7$ at 5 minute & & \\
\hline Proposed optimal GWG & Weight in $\mathrm{kg}$ & & & \\
\hline \multicolumn{5}{|l|}{ BMI categories $\left(\mathrm{kg} / \mathrm{m}^{2}\right)$} \\
\hline Underweight $<18.5$ & $12.5-18$ & $4-10^{*}$ & 8- 25 & $19.5(12.9 \text { to } 23.9)^{* * *}$ \\
\hline Normal weight 18.5-24.9 & $11.5-16$ & $2-10^{*}$ & $2-18$ & $13.7(7.7 \text { to } 18.8)^{* *}$ \\
\hline Overweight 24.9-29.9 & $7-11.5$ & $<9$ & -7 to 12 & $7.9(2.6 \text { to } 14.0)^{* *}$ \\
\hline Obese $\geq 30$ & $5-9.0$ & $<6$ & -15 to 2 & $1.8(-5.0 \text { to } 7.0)^{* *}$ \\
\hline
\end{tabular}

* BMI cutoff of 20.

** BMI cutoff of 18.5 to $<23$ for normal weight; 23 to $<27.5$ for overweight; $\geq 27.5$ for obese.

*** numbers in paraenthesis represent the lower and upper markings of the GWG range for which aggregated risk of composite adverse outcome does not exceed a 5\% increase from the lowest aggregated risk. 
Study ${ }^{42}$ evaluated the risk of several maternal and infant outcomes with a GWG outside of the guidelines in data on more than 50,000 women. There was an increased risk of macrosomia, preeclampsia and emergency caesarean section in the normal weight and overweight groups who exceeded GWG recommendation. Excess GWG across all weight gain groups resulted in postpartum weight retention of $>2 \mathrm{~kg}$ at 18 months.

The IOM guidelines have been criticized for their lack of global utility, given that they are based on mostly observational studies from developed Western countries. Their use of WHO BMI cut points are not specific for Asian women. With this is mind, $\mathrm{Ee}^{56}$ et al used the WHO BMI cut points for Asian women and created new optimal GWG recommendations in their multiethnic Singapore cohort (-Table 2). Of interest, optimal GWG in underweight and obese women was outside the IOM range.

While IOM recommendations currently guide practice in many countries, a recent study has shown significant variation in practice internationally in terms of policies on $\mathrm{GWG}^{57}$ To clearly define healthy GWG, the 2009 IOM guidelines need to be validated in the current setting of higher maternal BMI's and greater rates of GWG. Systematic review, meta-analysis and further research is needed addressing adverse outcomes across diverse multi-ethnic populations.

\section{Lifestyle Interventions and the Need for More Research}

Prevention of obesity is important for all lifelong. In this context, targeting pregnant women in prevention of weight gain is important as i) there are significant reproductive implications of obesity ii) many women now exceed international GWG recommendations ${ }^{11,13,58,59}$ and $\sim 2-5 \mathrm{~kg}$ are retained per pregnancy ${ }^{13,60}$ iii) pregnancy offers a defined life stage for women captured in our existing health system with enablers for lifestyle change iv) healthy lifestyle change in pregnancy improves outcomes $v$ ) weight gain during pregnancy contributes significantly to maternal obesity with potential for long term health benefits vi) women influence family lifestyle with maternal lifestyle changes having broader implications for families and communities.

Lifestyle interventions in pregnancy focusing on improving dietary intake and physical activity has been shown in a comprehensive systematic review of 7278 women to prevent excess GWG gain. Overall, there was $1.42 \mathrm{~kg}$ less weight gained (95\% confidence interval 0.95 to $1.89 \mathrm{~kg}$ ) and lifestyle intervention reduced preeclampsia (OR 0.39 - 0.74) and shoulder dystocia (OR 0.39), with a trend to reduced GDM (OR 0.78, CI 0.57-1.08). ${ }^{21}$ Lifestyle intervention do not appear to impact on birth weight, or have safety concerns. ${ }^{21}$ Monitoring maternal weight alone is ineffective, but improves efficacy when used in combination with interventions. ${ }^{61}$

There are many effective lifestyle interventions in pregnancy. The Healthy Lifestyle Program for women (HeLP-her) is one example of an effective intervention. ${ }^{21}$ The HeLP-her program is an evidence-based self-management weight gain prevention intervention initially targeting reproductive aged non pregnant women and published in the BMJ. ${ }^{62}$ Now trialled across different urban and metropolitan settings and populations, it has demonstrated efficacy in over 1000 women including women in pregnancy. ${ }^{10,63,64} \mathrm{HeLP}$-her has significant evidence of efficacy for weight gain prevention and is designed for implementation as a low cost pragmatic simple intervention that leverages off self-management and is integrated into routine antenatal care. ${ }^{6}$ It involves simple dietary and activity messages, self-management, behavioral strategies such as goal setting, problem solving, relapse prevention, self-monitoring, phone coaching and SMS reminder messages ${ }^{65}$ shown to support small lifestyle behavior changes and effective weight gain prevention. ${ }^{66,67}$ Diet messages follow national guidelines and include increased unprocessed grains, fruits and vegetables. ${ }^{10,21,64} \mathrm{HeLP}$-her has now been adapted to target limiting excess GWG, promoting postpartum weight loss and preventing type II diabetes in women with a history of GDM. This intervention is being trialled in large scale implementation research across lowmid socioeconomic, multiethnic countries in an internationally funded RCT of 1600 women in resource poor settings.

The UPBEAT study focused solely on obese women $\left(n=1555\right.$, mean BMI $\left.36.3 \mathrm{~kg} / \mathrm{m}^{2}\right)$, with the primary outcomes of maternal diagnosis of GDM and reduction of LGA. The intervention was relatively intensive with 8 , mainly group sessions combining behavioral components, dietary and physical activity advice. GWG and skinfold thickness were lower in the intervention group, although results were modest with $0.55 \mathrm{~kg}(95 \% \mathrm{CI}-1.08$ to -0.02$)$ less GWG in the intervention group, and no maternal or neonatal benefits demonstrated. ${ }^{68,69}$

The LIMIT study in Australia again had primary outcomes focused on reduction in neonatal complications, with the primary endpoint being reduction in LGA, rather than prevention of weight gain alone. Here, 2212 overweight and obese women were randomized to standard care with or without an additional lifestyle intervention. The intervention was delivered by a dietitian and was not integrated with routine antenatal care. ${ }^{70}$ This study did not show differences in the primary endpoint, but did show a reduction in babies born over 4000 gms. $^{70}$ These results are consistent with the majority of the literature in this area, which shows a failure to impact significantly on birth weight. ${ }^{21}$

Overall, antenatal lifestyle interventions, prevent excess GWG and offer important obesity prevention opportunities at a vital life stage, when women are engaged with the health system, ${ }^{6}$ yet they do not significantly alter birth weights and appear to have limited impact on neonatal outcomes.

Although systematic reviews had identified beneficial effects of mainly diet based, physical activity based and mixed interventions, ${ }^{21}$ findings are limited by the variation in the characteristics of the population, intervention and outcomes. The effects of lifestyle interventions on various groups of women based on BMI category, age, ethnicity, parity and risk status in pregnancy is not known. These questions cannot be answered from published aggregate data, as patient-level information is not available and subgroup effects ('treatment-covariate interactions') are usually not reported in 
sufficient detail. However, these gaps in evidence can be addressed by meta-analysis of individual participant data IPD, where the raw patient-level data are obtained and synthesized across trials.

The International Weight Management in Pregnancy (i-WIP) individual patient data (IPD) collaborative network is funded by the UK National Institute for Health Research (NIHR) to assess differential weight management interventions in pregnancy by BMI, age, ethnicity, parity and underlying medical conditions on a) maternal weight and b) composite pregnancy outcome of maternal and fetal complications. $^{30}$ The Network also aims to quantify the relationship between the amount of weight gained in pregnancy and the risk of adverse maternal and fetal outcomes for normal weight, overweight and obese women. The i-WIP Network comprises 36 principal investigators from 17 countries, and comprises of obstetricians, physicians, nutritionists, physiotherapists, researchers, dieticians, exercise physiologists, midwives, nurses and consumers involved in the evaluation of diet and physical activity on GWG and other complications in pregnancy.

The findings of the i-WIP initiative will soon enable us to clearly define the efficacy of lifestyle interventions in pregnancy to prevent excess GWG and obesity. It will also provide clarity on maternal (e.g., GDM, preeclampsia) benefits. Remaining clinical and research gaps which should be addressed in the i-WIP work, include the most effective components of lifestyle interventions, optimal delivery modes and a cost benefit analyses. ${ }^{30}$ This important information will inform implementation (the next vital step) and scale up of healthy lifestyle interventions to target the broader population of pregnant women outside those in randomized controlled trials.

In considering implementation of lifestyle interventions into routine antenatal care, barriers need to be addressed. Misperceptions around healthy weight among health professionals and women need to be redressed. For example, it is estimated that less than $16 \%$ of obese pregnant women identify as obese. ${ }^{71}$ Inadequate weight monitoring in routine care and disparities in medically advised GWG targets also needs to be rectified. ${ }^{72}$ Only $4 \%$ of obstetricians and midwives accurately identified IOM GWG recommendations, ${ }^{26}$ only $25-30 \%$ suggested weight targets; only $1 \%$ base targets on IOM guidelines and $\sim 70 \%$ reported inadequate training in lifestyle behavior change methods. ${ }^{26}$ In a midwifery survey, provision of lifestyle advice by midwives was limited and interventions to assist women and staff in developing skills to aid this intervention provision were lacking. ${ }^{73}$

Alongside identified barriers, implementation gaps in prior interventions include failure to partner to establish problems, engage stakeholders, address barriers and enablers, use implementation informed study design; expand beyond single institutions, as well as inconsistent designs, poor or unreported recruitment. There has been a lack of focus on normal and overweight women at highest risk of additional weight retention postpartum, failure to use theoretical frameworks, apply evidence based components, integrate into routine antenatal care and to provide implementation resources for health services and for health professionals. Finally there has also been limited postpartum extension ${ }^{74}$ and lack of evaluation. Implementation research is now needed to address these barriers and gaps. We need to know how best to address health system and health professional factors including how to engage, train and support health professionals in lifestyle change. Ideally this will include integrating key lifestyle message prompts, weighing reminders and triggers when GWG is exceeded, into routine maternity care workflows, as this approach is known to increase application of clinical guidelines by 20 -fold compared with provision of guidelines alone. ${ }^{75}$

\section{Conclusion}

Overweight and obesity present a major and neglected public health burden. Reproductive aged women are a recognized high risk target group for weight gain and related complications. Excess GWG is a significant contributor to obesity in women which carries independent increased risks of adverse maternal and infant outcomes, including and not limited to caesarean section, increased birth weight, LGA and long term maternal and childhood obesity. Further research is required to assist in refining and optimizing GWG recommendations across different BMI categories and ethnic groups. Individually targeted antenatal lifestyle interventions effectively limit excess GWG, contributing to prevention of obesity in reproductive aged women. Specific maternal and neonatal pregnancy benefits of these interventions still require clarification. Optimal components of antenatal lifestyle interventions as well as the cost effectiveness of these interventions are currently being researched through an international individual patient data meta-analysis of lifestyle interventions in pregnancy that will guide practice and policy in this area. We then require pragmatic implementation strategies to scale up healthy lifestyle into routine antenatal care.

Ultimately, antenatal interventions need integration with prevention efforts across the life stages including in childhood and adolescence and preconception to prevent maternal weight related pregnancy complications. While ultimately healthy lifestyle is a matter of individual behavior change, individual interventions must extend beyond individual targeted initiatives to address societal and environmental factors and enable children, adolescents and women to have a healthier lifestyle and to prevent obesity and related complications.

\section{References}

1 Apovian CM, Aronne LJ, Bessesen DH, et al; Endocrine Society. Pharmacological management of obesity: an endocrine Society clinical practice guideline. J Clin Endocrinol Metab 2015;100(2): 342-362

2 Wadden TA, Butryn ML, Wilson C. Lifestyle modification for the management of obesity. Gastroenterology 2007;132(6):2226-2238

3 Hill JO. Understanding and addressing the epidemic of obesity: an energy balance perspective. Endocr Rev 2006;27(7):750-761

4 Lombard CB, Deeks AA, Teede HJ. A systematic review of interventions aimed at the prevention of weight gain in adults. Public Health Nutr 2009;12(11):2236-2246 
5 World Health Organisation 2013-2020 action plan for the global strategy for the prevention and control of noncommunicable diseases: prevent and control cardiovascular diseases, cancers, chronic respiratory diseases and diabetes. Geneva: WHO; 2014

6 Teede H, Harrison C, Lombard C, Boyle J, East C, Brown W. Case for Action proposal: Obesity prevention through preventing excess weight gain during pregnancy and postpartum. National Health and Medical Research Council; 2015:20wwwnhmrcgovau/research/research-translation/research-translation-faculty/.

Accessed May 10, 2016

7 Colditz GA, Willett WC, Stampfer MJ, et al. Weight as a risk factor for clinical diabetes in women. Am J Epidemiol 1990;132(3): 501-513

8 Willett WC, Manson JE, Stampfer MJ, et al. Weight, weight change, and coronary heart disease in women. Risk within the 'normal' weight range. JAMA 1995;273(6):461-465

9 Winett RA, Tate DF, Anderson ES, Wojcik JR, Winett SG. Long-term weight gain prevention: a theoretically based Internet approach Prev Med 2005;41(2):629-641

10 Harrison CL, Lombard CB, Strauss BJ, Teede HJ. Optimizing healthy gestational weight gain in women at high risk of gestational diabetes: a randomized controlled trial. Obesity (Silver Spring) 2013;21(5):904-909

11 Chu SY, Callaghan WM, Bish CL, D’Angelo D. Gestational weight gain by body mass index among US women delivering live births, 2004-2005: fueling future obesity. Am J Obstet Gynecol 2009; 200(3):271.e1-271.e7

12 Fraser A, Tilling K, Macdonald-Wallis C, et al. Associations of Gestational Weight Gain With Maternal Body Mass Index, Waist Circumference, and Blood Pressure Measured 16 Years After Pregnancy: The Avon Longitudinal Study of Parents and Children. Obstet Gynecol Surv 2011;66:599-600

13 Rasmussen K, Yaktine AL, Eds. Institute of Medicine and National Research Council Committee to Reexamine IOM Pregnancy Weight Guidelines. Weight Gain During Pregnancy: Reexamining the Guidelines. Washington DC: National Academic Press; 2009

14 Amorim AR, Rössner S, Neovius M, Lourenço PM, Linné Y. Does excess pregnancy weight gain constitute a major risk for increasing long-term BMI? Obesity (Silver Spring) 2007;15(5): 1278-1286

15 World Health Organisation. 2008-2013 action plan for the global strategy for the prevention and control of noncommunicable diseases: prevent and control cardiovascular diseases, cancers, chronic respiratory diseases and diabetes. Geneva: WHO; 2008

16 Cedergren MI. Optimal gestational weight gain for body mass index categories. Obstet Gynecol 2007;110(4):759-764

17 Black MH, Sacks DA, Xiang AH, Lawrence JM. The relative contribution of prepregnancy overweight and obesity, gestational weight gain, and IADPSG-defined gestational diabetes mellitus to fetal overgrowth. Diabetes Care 2013;36(1):56-62

18 Institute of Medicine/National Research Council. Committee to Reexamine IOM Pregnancy Weight Guidelines, Food and Nutrition Board and Board on Children, Youth, and Families. Weight gain during pregnancy: reexamining the guidelines. Washington, DC: National Academies Press; 2009

19 Dodd J, Thangaratinam S; i-WIP collaborative network. Researchers' position statement on tackling obesity in pregnancy: the International Weight Management in Pregnancy (i-WIP) collaboration pleads for public health intervention. BJOG 2016;123(2): 163-164

20 Skouteris H, Huang T, Millar L, et al. A systems approach to reducing maternal obesity: The Health in Preconception, Pregnancy and Postbirth (HIPPP) Collaborative. Aust N Z J Obstet Gynaecol 2015;55(4):397-400

21 Thangaratinam S, Rogozińska E, Jolly K, et al. Effects of interventions in pregnancy on maternal weight and obstetric outcomes: meta-analysis of randomised evidence. BMJ 2012;344: e2088-e2093
22 Muktabhant B, Lumbiganon P, Ngamjarus C, Dowswell T. Interventions for preventing excessive weight gain during pregnancy. Cochrane Database Syst Rev 2012;4:CD007145

23 Hill B, Skouteris H, Fuller-Tyszkiewicz M. Interventions designed to limit gestational weight gain: a systematic review of theory and meta-analysis of intervention components. Obes Rev 2013;14(6): 435-450

24 van der Pligt P, Willcox J, Hesketh KD, et al. Systematic review of lifestyle interventions to limit postpartum weight retention: implications for future opportunities to prevent maternal overweight and obesity following childbirth. Obes Rev 2013;14(10): 792-805

25 Biro MA, Cant R, Hall H, Bailey C, Sinni S, East C. How effectively do midwives manage the care of obese pregnant women? A crosssectional survey of Australian midwives. Women Birth 2013; 26(2):119-124

26 Stewart ZA, Wallace E, Allan C. Weight gain in pregnancy: a survey of current practices in a teaching hospital. Aust $\mathrm{N} \mathrm{Z} \mathrm{J} \mathrm{Obstet}$ Gynaecol 2012;52(2):208-210

27 Phelan S, Phipps MG, Abrams B, Darroch F, Schaffner A, Wing RR. Practitioner advice and gestational weight gain. J Womens Health (Larchmt) 2011;20(4):585-591

28 Arslan Ozkan I, Mete S. Pregnancy planning and antenatal health behaviour: findings from one maternity unit in Turkey. Midwifery 2010;26(3):338-347

29 Edvardsson K, Ivarsson A, Eurenius E, et al. Giving offspring a healthy start: parents' experiences of health promotion and lifestyle change during pregnancy and early parenthood. BMC Public Health 2011;11:936

30 Ruifrok AE, Rogozinska E, van Poppel MNM, et al; i-WIP (International Weight Management in Pregnancy) Collaborative Group. Study protocol: differential effects of diet and physical activity based interventions in pregnancy on maternal and fetal outcomes -individual patient data (IPD) meta-analysis and health economic evaluation. Syst Rev 2014;3:131-137

31 Opray N, Grivell RM, Deussen AR, Dodd JM. Directed preconception health programs and interventions for improving pregnancy outcomes for women who are overweight or obese. Cochrane Database Syst Rev 2015;7:CD010932

32 Kurukulasuriya LR, Stas S, Lastra G, Manrique C, Sowers JR. Hypertension in obesity. Med Clin North Am 2011;95(5):903-917

33 Reaven GM. Insulin resistance: the link between obesity and cardiovascular disease. Med Clin North Am 2011;95(5): 875-892

34 Mehra R, Redline S. Sleep apnea: a proinflammatory disorder that coaggregates with obesity. J Allergy Clin Immunol 2008;121(5): 1096-1102

35 Hillemeier MM, Downs DS, Feinberg ME, et al. Improving women's preconceptional health: findings from a randomized trial of the Strong Healthy Women intervention in the Central Pennsylvania women's health study. Womens Health Issues 2008;18(6, Suppl) S87-S96

36 Weisman CS, Hillemeier MM, Downs DS, et al. Improving women's preconceptional health: long-term effects of the Strong Healthy Women behavior change intervention in the central Pennsylvania Women's Health Study. Womens Health Issues 2011;21(4): 265-271

37 Weisman CS, Hillemeier MM, Downs DS, Chuang CH, Dyer AM. Preconception predictors of weight gain during pregnancy: prospective findings from the Central Pennsylvania Women's Health Study. Womens Health Issues 2010;20(2):126-132

38 National Institute for Health and Clinical Excellence (NICE). Dietary interventions and physical activity interventions for weight management before, during and after pregnancy. 2010, London, UK

39 Thangaratinam S, Jolly K. Obesity in pregnancy: a review of reviews on the effectiveness of interventions. BJOG 2010; 117(11):1309-1312 
40 Viswanathan M, Siega-Riz AM, Moos MK, et al. Outcomes of maternal weight gain. Evid Rep Technol Assess (Full Rep) 2008; (168):1-223

41 Margerison Zilko CE, Rehkopf D, Abrams B. Association of maternal gestational weight gain with short- and long-term maternal and child health outcomes. Am J Obstet Gynecol 2010;202(6):574. e1-574.e8

42 Haugen M, Brantsæter AL, Winkvist A, et al. Associations of prepregnancy body mass index and gestational weight gain with pregnancy outcome and postpartum weight retention: a prospective observational cohort study. BMC Pregnancy Childbirth 2014; 14:201-205

43 Nohr EA, Vaeth M, Baker JL, Sørensen Tla, Olsen J, Rasmussen KM. Combined associations of prepregnancy body mass index and gestational weight gain with the outcome of pregnancy. Am J Clin Nutr 2008;87(6):1750-1759

44 Li N, Liu E, Guo J, et al. Maternal prepregnancy body mass index and gestational weight gain on pregnancy outcomes. PLOS ONE 2013;8(12):e82310

45 Hedderson MM, Gunderson EP, Ferrara A. Gestational weight gain and risk of gestational diabetes mellitus. Obstet Gynecol 2010; 115(3):597-604

46 Yu Z, Han S, Zhu J, Sun X, Ji C, Guo X. Pre-pregnancy body mass index in relation to infant birth weight and offspring overweight/ obesity: a systematic review and meta-analysis. PLoS ONE 2013; 8(4):e61627

47 Rooney BL, Schauberger CW. Excess pregnancy weight gain and long-term obesity: one decade later. Obstet Gynecol 2002;100(2): 245-252

48 Fraser A, Tilling K, Macdonald-Wallis C, et al. Associations of gestational weight gain with maternal body mass index, waist circumference, and blood pressure measured $16 \mathrm{y}$ after pregnancy: the Avon Longitudinal Study of Parents and Children (ALSPAC). Am J Clin Nutr 2011;93(6):1285-1292

49 Rooney BL, Schauberger CW, Mathiason MA. Impact of perinatal weight change on long-term obesity and obesity-related illnesses. Obstet Gynecol 2005;106(6):1349-1356

50 Oken E, Kleinman KP, Belfort MB, Hammitt JK, Gillman MW. Associations of gestational weight gain with short- and longerterm maternal and child health outcomes. Am J Epidemiol 2009; 170(2):173-180

51 Gaillard R, Welten M, Oddy WH, et al. Associations of maternal prepregnancy body mass index and gestational weight gain with cardio-metabolic risk factors in adolescent offspring: a prospective cohort study. BJOG 2016;123(2):207-216

52 Hrolfsdottir L, Rytter D, Olsen SF, et al. Gestational weight gain in normal weight women and offspring cardio-metabolic risk factors at 20 years of age. Int J Obes 2015;39(4):671-676

53 Shi P, Yang W, Yu Q et al. Overweight, gestational weight gain and elevated fasting plasma glucose and their association with macrosomia in chinese pregnant women. Matern Child Health J 2014; 18(1):10-15

54 Potti S, Sliwinski CS, Jain NJ, Dandolu V. Obstetric outcomes in normal weight and obese women in relation to gestational weight gain: comparison between Institute of Medicine guidelines and Cedergren criteria. Am J Perinatol 2010;27(5):415-420

55 Beyerlein A, Schiessl B, Lack N, von Kries R. Optimal gestational weight gain ranges for the avoidance of adverse birth weight outcomes: a novel approach. Am J Clin Nutr 2009;90(6):1552-1558

56 Ee TX, Allen JC Jr, Malhotra R, Koh H, Østbye T, Tan TC. Determining optimal gestational weight gain in a multiethnic Asian population. J Obstet Gynaecol Res 2014;40(4):1002-1008

57 Scott C, Andersen CT, Valdez N, et al. No global consensus: a crosssectional survey of maternal weight policies. BMC Pregnancy Childbirth 2014;14:167
58 Abrams B, Selvin S. Maternal weight gain pattern and birth weight. Obstet Gynecol 1995;86(2):163-169

59 Harrison CL, Lombard CB, Teede HJ. Understanding health behaviours in a cohort of pregnant women at risk of gestational diabetes mellitus: an observational study. BJOG 2012;119(6):731-738

60 Women's Weight: Findings From the Australian Longitudinal Study on Women's Health. . Australian Government Department of Health \& Ageing. June 2007. at http://www.alswh.org.au/Reports/OtherReportsPDF/ALSWHMajorReportB310707_final.pdf. Accessed May 10, 2016

61 Jeffries K, Shub A, Walker SP, Hiscock R, Permezel M. Reducing excessive weight gain in pregnancy: a randomised controlled trial. Med J Aust 2009;191(8):429-433

62 Lombard C, Deeks A, Jolley D, Ball K, Teede H. A low intensity, community based lifestyle programme to prevent weight gain in women with young children: cluster randomised controlled trial. BMJ 2010;341:c3215

63 Lombard CB, Harrison CL, Kozica SL, Zoungas S, Keating C, Teede HJ. Effectiveness and implementation of an obesity prevention intervention: the HeLP-her Rural cluster randomised controlled trial. BMC Public Health 2014; 14:608

64 Harrison CL, Lombard CB, Teede HJ. Limiting postpartum weight retention through early antenatal intervention: the HeLP-her randomised controlled trial. Int J Behav Nutr Phys Act 2014; $11: 134$

65 Stephens J, Allen J. Mobile phone interventions to increase physical activity and reduce weight: a systematic review. J Cardiovasc Nurs 2013;28(4):320-329

66 Artinian NT, Fletcher GF, Mozaffarian D, et al; American Heart Association Prevention Committee of the Council on Cardiovascular Nursing. Interventions to promote physical activity and dietary lifestyle changes for cardiovascular risk factor reduction in adults: a scientific statement from the American Heart Association. Circulation 2010;122(4):406-441

67 Wadden TA, Butryn ML, Byrne KJ. Efficacy of lifestyle modification for long-term weight control. Obes Res 2004;12(Suppl): 151S-162S

68 Briley AL, Barr S, Badger S, et al. A complex intervention to improve pregnancy outcome in obese women; the UPBEAT randomised controlled trial. BMC Pregnancy Childbirth 2014;14:74

69 Poston L, Bell R, Croker H, et al; UPBEAT Trial Consortium. Effect of a behavioural intervention in obese pregnant women (the UPBEAT study): a multicentre, randomised controlled trial. Lancet Diabetes Endocrinol 2015;3(10):767-777

70 Dodd JM, Turnbull D, McPhee AJ, et al; LIMIT Randomised Trial Group. Antenatal lifestyle advice for women who are overweight or obese: LIMIT randomised trial. BMJ 2014;348: g1285

71 Callaway LK, O'Callaghan MJ, McIntyre HD. Barriers to addressing overweight and obesity before conception. Med J Aust 2009; 191(8):425-428

72 Cogswell ME, Scanlon KS, Fein SB, Schieve LA. Medically advised, mother's personal target, and actual weight gain during pregnancy. Obstet Gynecol 1999;94(4):616-622

73 Power ML, Cogswell ME, Schulkin J. Obesity prevention and treatment practices of U.S. obstetrician-gynecologists. Obstet Gynecol 2006;108(4):961-968

74 Oteng-Ntim E, Varma R, Croker H, Poston L, Doyle P. Lifestyle interventions for overweight and obese pregnant women to improve pregnancy outcome: systematic review and meta-analysis. BMC Med 2012;10:47

75 Kawamoto K, Houlihan CA, Balas EA, Lobach DF. Improving clinical practice using clinical decision support systems: a systematic review of trials to identify features critical to success. BMJ 2005; 330(7494):765 